\title{
HUASTECA 700, VARIEDAD DE SOYA PARA EL SUR DE TAMAULIPAS, MÉXICO
}

\author{
HUASTECA 700, SOYBEAN VARIETY FOR SOUTHERN TAMAULIPAS, MEXICO
}

\author{
Nicolás Maldonado-Moreno, Guillermo Ascencio-Luciano \\ y Julio C. García-Rodríguez*
}

Instituto Nacional de Investigaciones Forestales, Agrícolas y Pecuarias, Campo Experimental Las Hustecas, Altamira, Tamaulipas, México.

*Autor para correspondencia (garcia.juliocesar@inifap.gob.mx)

En el sur de Tamaulipas, México, el cultivo de la soya [Glycine max (L.) Merrill] se practica en condiciones de temporal durante el ciclo primavera-verano desde hace 40 años. La superficie sembrada con soya ha variado entre 90 mil y 124 mil hectáreas en los últimos años, con rendimientos promedio que fluctúan de 0.82 a $1.80 \mathrm{t}$ ha 1 (SIAP, 2018), lo cual depende del nivel tecnológico que aplican los productores y de las condiciones climatológicas prevalecientes durante el ciclo del cultivo. Los rendimientos de grano se ven limitados por la falta de humedad durante la etapa reproductiva de la soya y por la sensibilidad de las variedades al fotoperiodo corto, principalmente cuando la fecha de siembra se retrasa debido a deficiencias hídricas. Por lo anterior, el enfoque del mejoramiento genético de la soya en el trópico mexicano se centra en la generación de genotipos con 45 días a floración como mínimo, para no mermar el rendimiento, y que el periodo entre las etapas fenológicas R4 (vaina completa) y R7 (inicio de madurez fisiológica), según el perfil de Fehr y Caviness (1977), coincida con la época de mayor probabilidad de lluvias, sin dejar de considerar las características de alta productividad, baja sensibilidad al fotoperiodo corto, tolerancia a sequía, enfermedades foliares más comunes y contenido de proteína y aceite en la semilla.

Con base en los criterios mencionados se generó la variedad de soya Huasteca 700, a partir de la hibridación de los genotipos Santa Rosa × F81-5517 que se realizó en el Campo Experimental Las Huastecas del Instituto Nacional de Investigaciones Forestales, Agrícolas y Pecuarias (INIFAP) en el ciclo primavera-verano 1997. Los progenitores pertenecen al grupo de madurez IX, con adaptación a latitudes tropicales. Santa Rosa es una variedad que se introdujo de Brasil en 1979; fue generada por el Instituto Agronómico de Campinas, Sao Paulo (Bonato y Bonato, 2002), presenta valores altos en los componentes del rendimiento y se adapta a las condiciones cálido-húmedas del trópico. La línea F81-5517 se introdujo de Estados Unidos de América en 1985; fue desarrollada por el Servicio de Investigación Agrícola del Departamento de Agricultura en Gainesville, Florida; es de baja sensibilidad al fotoperiodo corto y presenta resistencia moderada a las enfermedades causadas por Cercospora sojina, Peronospora manshurica y Septoria glycines, entre otras. La $F_{1}$ se obtuvo en el ciclo primavera-verano 1998 Las poblaciones segregantes $F_{2}$ a $F_{4}$ se avanzaron por el método de pedigrí modificado o descendencia por vaina simple (Fehr, 1993). En la $\mathrm{F}_{4}$ se seleccionaron 180 plantas individuales; posteriormente, se evaluó la progenie y la selección en masa se realizó en la $\mathrm{F}_{5^{\prime}}$ dando origen a la línea H02-1656, hoy Huasteca 700. En el ciclo primaveraverano 2003 se realizó la prueba preliminar de rendimiento. Las evaluaciones de rendimiento y sus componentes, así como de las características fenológicas en las etapas preliminar, regional y trópico húmedo se realizaron de 2004 a 2012. Una vez determinado el potencial productivo y los atributos agronómicos de la línea H02-1656 a nivel experimental, se realizó la validación con productores en el sur de Tamaulipas, durante los años 2014 y 2015.

Huasteca 700 es de porte alto (Figura 1), con baja sensibilidad al fotoperiodo corto y de hábito de crecimiento determinado, tipo arbusto (produce ramas). Su pubescencia es café, la hoja es mediana, con foliolos ovoides color verde medio, las flores son color violeta, la semilla es esférica, de color amarillo e hilio café. El peso de 100 semillas es de $14.4 \mathrm{~g}$, tiene vainas con dos y tres granos, presenta 53 días a R2 (floración completa), lo que le permite desarrollar el periodo de vaina completa a inicio de madurez (R4-R7) en la época de mayor probabilidad de lluvias (septiembre y octubre), y 119 días a R8 (madurez fisiológica). Su altura es de $93 \mathrm{~cm}$, logrando un crecimiento adecuado para que exprese su potencial de rendimiento cuando se siembra del 15 de junio al 20 de julio. Tiene buen nivel de resistencia al acame y al desgrane. Puede sembrarse en surcos de 76 a $80 \mathrm{~cm}$ de ancho o más 


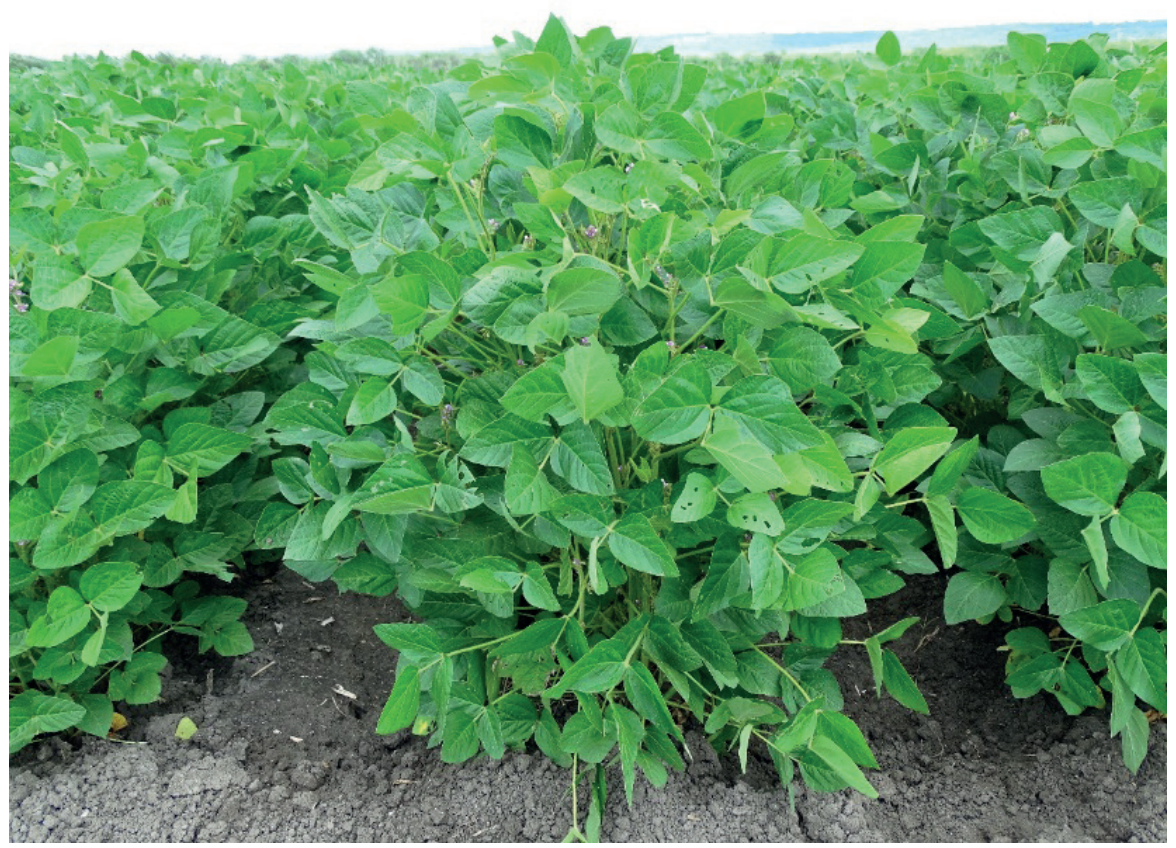

Figura 1. Estructura botánica de la variedad de soya Huasteca 700.

angostos, a una densidad de 250 mil a 300 mil plantas ha $^{-1}$. En cuanto a calidad de semilla, ésta tiene $23.3 \%$ de aceite y $35.6 \%$ de proteína, niveles muy cercanos a los que demanda la industria aceitera del país ( $20 \% \pm 2$ de aceite y de 38 a $40 \%$ de proteína).

Esta variedad es resistente a la enfermedad "ojo de rana" (Cercospora sojina), ya que al evaluar su respuesta a enfermedades presentó ausencia de síntomas o infecciones menores al $10 \%$ en el área foliar, de manera similar al comportamiento de Huasteca 400 (Maldonado et al., 2010). Es moderadamente susceptible a mildiú velloso (Peronospora manshurica), tiro de munición (Corynespora cassiicola) y antracnosis (Colletotrichum truncatum). En cuanto al ataque de insectos, tolera hasta $30 \%$ de defoliación en la etapa vegetativa sin que se afecte el rendimiento, de igual manera que Huasteca 400 (Maldonado et al., 2010); además, la preferencia por las principales larvas defoliadoras, el gusano terciopelo (Anticarsia gemmatalis) y el gusano falso medidor de la soya (Pseudoplusia includens), no es mayor en comparación con las variedades comerciales.

Varios índices de resistencia a sequía señalan que Huasteca 700 presenta buen nivel de tolerancia al estrés en la etapa reproductiva. La evaluación de García-Rodríguez et al. (2017) mostró que Huasteca 700 sobresalió en los índices de tolerancia al estrés (0.79), en la productividad media (1281) y en la productividad media geométrica (1132), cuando se comparó con las variedades Huasteca
200 y Huasteca 400, cuyos valores de los índices fueron 0.49, 997 y 898 respectivamente para el caso de la primera, y $0.85,989$ y 946 respectivamente para el caso de la segunda.

Las evaluaciones de rendimiento en los ensayos uniformes regionales y a nivel trópico húmedo bajo condiciones de temporal indicaron que Huasteca 700 rindió en promedio $2975 \mathrm{~kg} \mathrm{ha}^{-1}$ en el periodo de 2009 a 2012 en el sur de Tamaulipas, mientras que el rendimiento del testigo Huasteca 200 fue de $2492 \mathrm{~kg} \mathrm{ha}^{-1}$ (Cuadro 1). Los resultados obtenidos en parcelas semi-comerciales en 2014 y 2015 (Cuadro 2) mostraron que Huasteca 700 superó de 3.5 a 68.8 \% el rendimiento de Huasteca 400, utilizada como testigo.

Huasteca 700 tiene su mejor adaptación en el sur de Tamaulipas, principalmente en los municipios de Altamira, González y Mante, cuyo clima es cálido húmedo y subhúmedo, con precipitación anual de 800 a 1000 mm y temperatura media anual de 25 a $27^{\circ} \mathrm{C}$. El fotoperiodo durante la época de siembra en el ciclo primavera-verano tiene una duración de 13:32 a 13:11 horas luz y la mayor parte de los suelos son vertisoles. No obstante, puede sembrarse en las áreas productoras de soya de las regiones oriente de San Luis Potosí (municipios de Ébano y Tamuín) y norte de Veracruz (municipio de Pánuco), ya que las condiciones de clima y suelo son similares a las del sur de Tamaulipas. 
Cuadro 1. Rendimiento de la variedad Huasteca 700 en comparación con la variedad testigo Huasteca 200 bajo condiciones experimentales en el sur de Tamaulipas.

\begin{tabular}{|c|c|c|c|}
\hline \multirow{2}{*}{ Fecha de siembra } & Huasteca 700 & Huasteca 200 & \multirow{2}{*}{$\%$ relativo al testigo } \\
\hline & \multicolumn{2}{|c|}{$\left(\mathrm{kg} \mathrm{ha}^{-1}\right)$} & \\
\hline 08/09/2009 & 2379 & 2259 & +5.3 \\
\hline 05/07/2010 & 2764 & 2367 & +16.7 \\
\hline 11/07/2011 & 2647 & 2145 & +23.4 \\
\hline $27 / 06 / 2012$ & 4112 & 3199 & +28.5 \\
\hline Promedio & 2975 & 2492 & +19.3 \\
\hline
\end{tabular}

Cuadro 2. Rendimiento de la variedad Huasteca 700 a nivel semi-comercial en el sur de Tamaulipas, en comparación con la variedad testigo Huasteca 400 .

\begin{tabular}{lcccc}
\hline \multirow{2}{*}{ Año } & Localidad & Huasteca 700 & Huasteca 400 & \multirow{2}{*}{ \% relativo al testigo } \\
\cline { 3 - 4 } & Rancho El Peterón, Altamira & 2764 & 2671 & +3.5 \\
2014 & Rancho El Peterón, Altamira & 1062 & 1018 & +4.3 \\
2015 & Ejido Esteros, Altamira & 3029 & 1871 & +61.9 \\
2014 & Ejido Esteros, Altamira & 1433 & 849 & +68.8 \\
2015 & Ejido Cervantes, Altamira & 1748 & 1630 & +7.2 \\
2014 & & 2007 & 1607 & +24.8 \\
\hline
\end{tabular}

La variedad Huasteca 700 se encuentra protegida intelectualmente mediante el título de obtentor 1748 emitido a favor del INIFAP por el Servicio Nacional de Inspección y Certificación de Semillas de la SAGARPA. En 2017 se obtuvo el registro definitivo SOY-016-230217 por el organismo mencionado. Los interesados en la producción de semilla registrada y certificada de la variedad pueden acudir al Campo Experimental Las Huastecas del INIFAP donde se dispone de semilla básica. La semilla certificada puede conseguirse en diversas empresas productoras de semilla de soya en la región Huasteca.

\section{BIBLIOGRAFÍA}

Bonato E. R. e A. L. V. Bonato (2002) Cultivares que Fizeram a História da Soja no Rio Grande do Sul. Empresa Brasileira de Pesquisa Agropecuária-Trigo. Passo Fundo, Rio Grande do Sul, Brasil. $105 \mathrm{p}$

Fehr W. R. (1993) Principles of Cultivar Development: Theory and Technique. Iowa State University Press. Ames, lowa. 536 p.

Fehr W. R. and C. E. Caviness (1977) Stages of soybean development. Special Report 80. Iowa Satate University. Ames, lowa. 11 p.

García-Rodríguez J. C., N. Maldonado-Moreno y G. Ascencio-Luciano (2017) Caracterización de la tolerancia a sequía en soya [Glycine max (L.) Merr.] a través del esquema riego-sequía. Revista Biológico Agropecuaria Tuxpan 6:1678-1686.

Maldonado M. N., G. Ascencio L. y H. R. Gill L. (2010) Huasteca 400, nueva variedad de soya para el sur de Tamaulipas, oriente de San Luis Potosí y norte de Veracruz. Revista Mexicana de Ciencias Agrícolas 1:687-692.

SIAP, Servicio de Información Agropecuaria y Pesquera (2018) Producción agrícola. Servicio de Información Agropecuaria y Pesquera, SAGARPA. Ciudad de México. https://nube.siap.gob.mx/cierreagricola/ (Diciembre 2018). 
